\title{
Utilización de los servicios de hospitalización, consulta externa y emergencia, por adultos mayores y demenor edad, en el Hospital Nacional Cayetano Heredia: $1990-1997$.
}

CHIGNE VERÁSTEGUI Oscar, VARELA PINEDOLuis*,CHÁVEZJIMENOHelver** y SANDOVAL BERROCALLuis *** SUMMARY

Objective: To determine the significative utilization of health services by older people attended at Hospital Nacional Cayetano Heredia between 1990-1997, by means to compare these instrument and their characteristics, between older and younger patients. Material and methods: In this retrospective study, 14-year-old and older patients attended in the hospitalization service in 1990-1996 and outpatient and emergency services during 1997 were included. Information was elicited from egress files and statistical consolidates and was tabulated for age group, to asses comparative effects between older and younger people of 60 years old. Results: Hospitalization: Older people made up $28.11 \%$ of attended patients $[p<0.01]$. In the 1990-1996 period, there was an increase of older people $(+19.4 \%)$ and a decrease in younger ones $(-6.41 \%)\left[R^{2}=\mathbf{0 . 5 7 6 8}\right]$. In the older group, infections $(33.9 \%)$ and cardiovascular diseases $(32.9 \%)$ predominated. Geriatric mortality was relevant $(12.8 \%)[p<0.01]$. In the 1990-1996 period, there was a decrease of geriatric mortality $(-25 \%)$ with no variation in the younger group. $\left[R^{2}=0.4045\right]$. Outpatient service: The older group made up $16.76 \%$ of the attended patients, with female almost doubling in number the male patients [p<0.01]. The older group predominated over the younger ones and over those older who were attended only once $[p<0.01]$. The return rate end the ratio of attentions/attended people was in favor of the older group. The main geriatric outpatient morbidities were cardiovascular $(20.14 \%)$. Emergency : The older group made up $6.2 \%$ of the attended patients. The traumatologic entities were the most important in both age groups, with no evidence of significative differences. Conclusions: The utilization of hospitalization and outpatient services made by older people was significative relative to the younger group. ( Rev Med Hered 1999; 10: 111-118).

KEY WORDS: Geriatrics, old adult, health services.

\section{RESUMEN}

Objetivo: Determinar la significativa utilización de servicios de salud por los adultos mayores usuarios del Hospital Nacional Cayetano Heredia entre 1990-97, mediante la comparación de dicho instrumento y sus características, entre pacientes geriátricos y de menor edad. Material y métodos: En este estudio retrospectivo, se incluyeron pacientes de 14 años y más, atendidos en hospitalización entre 1990-96, y en consulta externa y emergencia durante 1997. La información fue relevada de libros de alta y consolidados estadísticos, siendo tabulada por grupos de edad, para efectos comparativos entre pacientes mayores y menores de 60 años. Resultados: Hospitalización: los adultos mayores constituyeron 28.11\% de los atendidos [p<0.01]. Entre 1990-96 hubo

Departamento de Medicina, servicio de Geriatría Hospital Nacional Cayetano Heredia. Universidad Peruana Cayetano Heredia. Lima, Perú.

** Departamento de Medicina, servicio de Geriatría Hospital Nacional Cayetano Heredia. Lima, Perú.

*** $\quad$ Médico Geriatra, Clínica San Pablo.
} 
incremento de pacientes geriátricos $(+19.4 \%)$ y disminución en los menores $(-6.41 \%)\left[\mathrm{R}^{2}=0.5768\right]$. A nivel geriátrico predominaron infecciones (33.9\%) y enfermedades del aparato circulatorio (32.9\%). La mortalidad geriátrica fue predominante $(12.8 \%)[\mathrm{p}<0.01]$. Entre 1990-96 hubo decrecimiento de mortalidad geriátrica $(-25 \%)$, sin observar cambios en los menores $\left[\mathrm{R}^{2}=0.4045\right]$. Consulta externa: los adultos mayores constituyeron $16.76 \%$ de los atendidos, con una población femenina que casi duplica a los varones [p<0.01]. Los gerontes continuadores predominaron sobre los menores y aquellos gerontes que consultaron sólo una vez [p<0.01]. La tasa de retorno y razón de atenciones/atendidos favoreció a los adultos mayores. La morbilidad geriátrica ambulatoria predominante fueron las cardiovasculares (20.14\%). Emergencia: los pacientes geriátricos constituyeron 6.2\% de los atendidos. Las entidades traumatológicas fueron predominantes en ambos grupos de edad, sin evidenciar diferencias significativas. Conclusiones: La utilización de los servicios de hospitalización y consulta externa realizada por los pacientes geriátricos, fue significativa en relación a los más jóvenes. ( Rev Med Hered 1999; 10: 111-118 ).

PALABRAS CLAVE: Adulto mayor, geronte, geriatría, servicios de salud, geriátrico.

\section{INTRODUCCION}

La continua tendencia mundial hacia una creciente mayor esperanza de vida ha consolidado el fenómeno de envejecimiento demográfico en los últimos años, con efectos impredecibles en las estructuras sociales, económicas, políticas, sanitarias, entre otras, afectando incluso la vida del mismo adulto mayor y su comunidad $(1,2)$. En 1990, la población adulta mayor de 60 años en el mundo, se calculaba en $9.2 \%$ de la población total y se estima en $14.2 \%$ en el año 2025. Según la proyección nacional de 1998, los de 60 años y más componen el 6.9\% del total de habitantes, estimándose en 12.6\% para el 2025 (3). A nivel internacional existen actitudes reiterativas a la constitución de políticas de atención geriátrica integral, orientadas al desempeño multidimensional de roles que superan el campo de interés médico tradicional, dando al adulto mayor un papel activo en el desempeño y optimización de la sociedad $(4,5,6)$.

Nuestro medio adolece de una evidente insuficiencia de atención integral, infraestructura inadecuada e información representativa sobre el perfil del adulto mayor, siendo incapaces de realizar medidas adecuadas a fin de alcanzar un grado aceptable de atención geriátrica $(7,8,9)$. Es apremiante, por tanto, la apertura del conocimiento disponible, mediante instrumentos que expresen el comportamiento de los diferentes parámetros asociados a la atención médica del adulto mayor $(10,11)$.

La demanda, definida como la cantidad de servicios de salud que necesitan ser atendidos en la población, se diferencia de la utilización de servicios (Demanda atendida), que viene a ser el reflejo de las necesidades percibidas y expresadas por los pacientes, las cuales son efectivamente atendidas por las entidades prestadoras de servicios, siendo factible su cuantificación a través de los registros continuos de producción de actividades de salud, y constituyendo un instrumento esencial en la búsqueda de información adecuada que sustente la configuración final de estrategias y políticas de atención.geriátrica $(12,13,14)$. En tal sentido, el propósito del presente estudio fue determinar si existe una significativa utilización de servicios de salud por la población geriátrica en los servicios de hospitalización, consulta externa y emergencia del Hospital Nacional Cayetano Heredia, mediante la comparación de dicho instrumento y sus características, entre los pacientes geriátricos y los pacientes de menor edad.

\section{MATERIAL Y METODOS}

Se realizó un estudio retrospectivo, cuyo universo estuvo constituido por pacientes de 14 años y más, atendidos en el Hospital Nacional Cayetano Heredia durante el período 1990-97.

Los pacientes hospitalizados entre 1990-96, provenientes de los servicios de hospitalización del Departamento de Medicina y del Departamento de Enfermedades Transmisibles y Dermatológicas, fueron obtenidos de los libros de alta correspondientes, excluyéndose 89 egresos $(0.7 \%)$ por carecer de datos completos.

Los pacientes atendidos en consulta externa, provenientes de los consultorios de Medicina, Infectología y Geriatría y en emergencia durante 1997, fueron obtenidos de las hojas de registro informático de consulta externa y consolidados de morbilidad, provenientes del Departamento de Estadística del Hospital Nacional Cayetano Heredia.

Es oportuno aclarar la necesidad de haber realizado distribuciones porcentuales independientes en ambas poblaciones de estudio, es decir, mayores y menores de 60 años, a fin de conseguir un adecuado método de comparación estadística en el contexto de las diferentes variables e indicadores descritos (15). Además, las distribuciones de ambos grupos, en términos porcentuales e independientes una de otra, fueron establecidas según un punto de corte establecido por nosotros y basado en estudios precedentes. Así, si bien el envejecimiento es un proceso paulatino cuyo inicio cronológico no ha sido 
Figura $N^{\circ} 1$. Distribución de hospitalización por años, según grupo de edad: 1990-96.

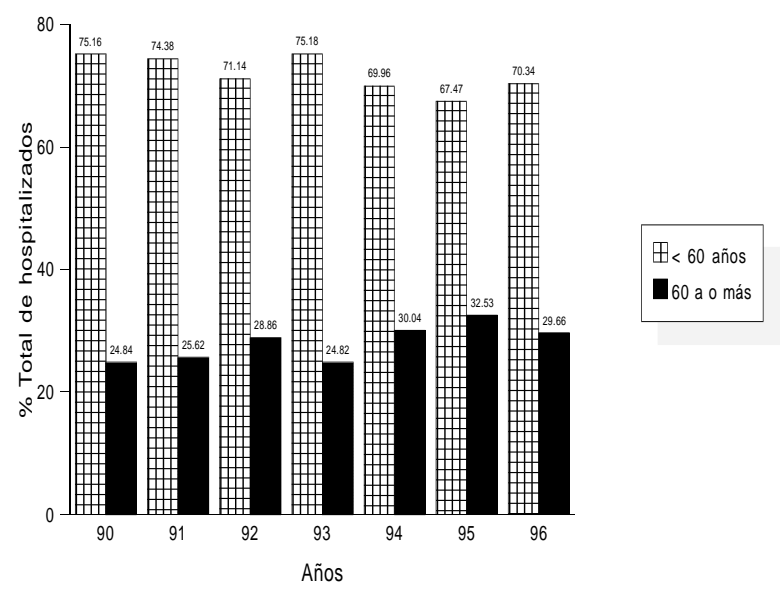

Figura $\mathrm{N}^{\circ} 2$. Promedio de Permanencia hospitalaria por grupos de edad: 1990-96.

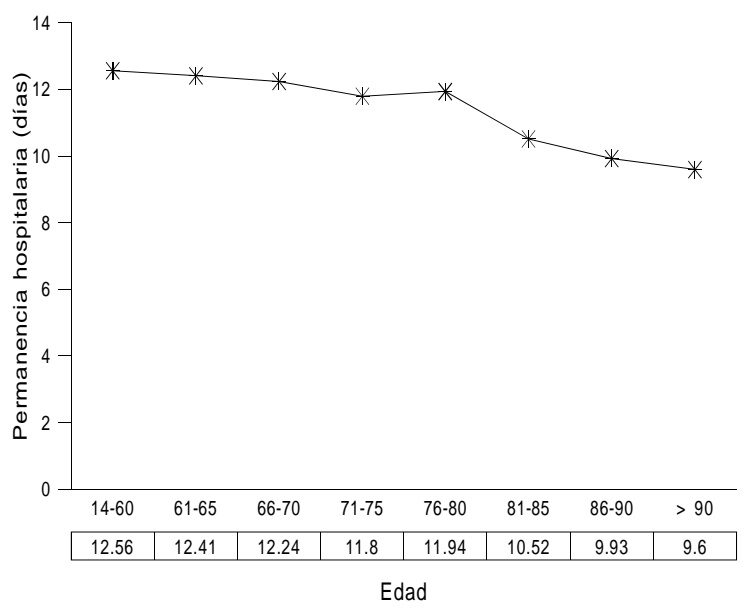

Figura $N^{\circ}$ 3. Permanencia hospitalaria por años, según edad. 1990-96

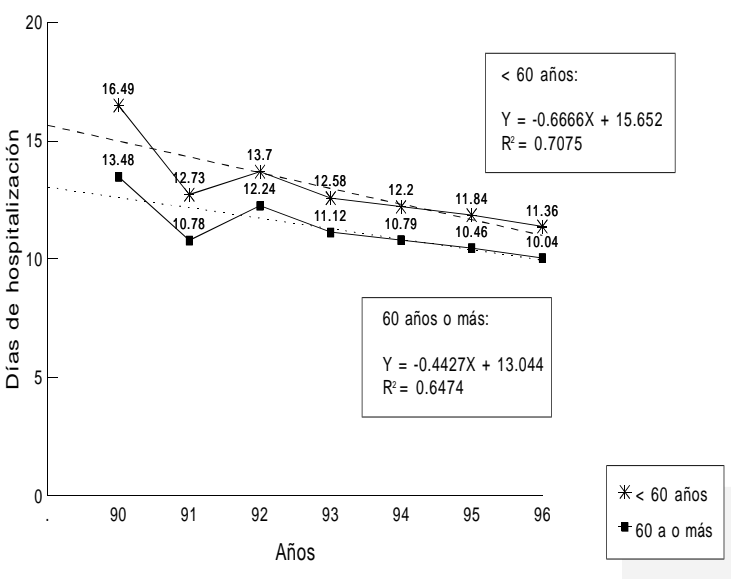

definido internacionalmente, el presente estudio utiliza el límite inferior de 60 años, adoptado en la Asamblea Mundial del Envejecimiento realizada en Viena y convocada por la ONU, como el más adecuado a nuestra realidad local $(15,16)$.

Se realizaron pruebas de media aritmética, pruebas de hipótesis, pruebas de Chi-cuadrado y método de regresión lineal y se utilizó el programa estadístico SPSS versión 7.5.

\section{RESULTADOS}

\section{Hospitalización:}

Durante 1990-96 fueron hospitalizados 12686 pacientes de 14 años de edad o más. Del total de internamientos, 3566 pacientes $(28.11 \%)$ correspondieron a pacientes de 60 años de edad o más. La mayor concentración geriátrica se registró en el grupo de 60 a 69 años, con $43.2 \%$ del total de adultos mayores hospitalizados. La edad geriátrica promedio fue de 73.49 años (rango:60 a 106 años). Además, la distribución hospitalaria entre 1990-96, reveló un crecimiento progresivo y significativo de $+19.4 \%$ en los adultos mayores y $-6 \%$ en los menores de 60 años $\left[\mathrm{R}^{2}=0.5748\right]$ (Figura $\mathrm{N}^{\circ} 1$ ).

Los varones constituyeron el $60.55 \%$ y $51.82 \%$ de menores y mayores de 60 años, respectivamente. La distribución por grupos de edad mostró una predominancia de mujeres mayores de 75 años, con $21 \%$ respecto al $18 \%$ de sus homólogos varones [ $\mathrm{p}<0.01]$. La edad geriátrica promedio de los varones hospitalizados fue de 72.9 años, frente a 74.12 años de las mujeres.

La permanencia hospitalaria para los mayores y menores de 60 años fue de 11.68 y 12.56 días, respectivamente. La distribución por grupos de edad mostró una disminución del promedio de permanencia hospitalaria conforme se avanza en edad (Figura $\mathrm{N}^{\circ} 2$ ). La variación del promedio total de permanencia hospitalaria entre 1990-96, significó una tendencia conjunta al decrecimiento de $-12 \%$ y $-28 \%$ para los mayores y menores de 60 años, respectivamente $\left[\mathrm{R}^{2}=0.6474\right]$ (Figura $\mathrm{N}^{\circ} 3$ ). Además, fue significativo el aumento del promedio de permanencia hospitalaria de decesos, a través de los años, equivalente a $+18 \%$ y $+26 \%$ para los mayores y menores de 60 años, respectivamente $\left[\mathrm{R}^{2}=0.5400\right]$ (Figura $\mathrm{N}^{\circ} 4$ ).

Las infecciones fueron las causas mas frecuentes de hospitalización en los pacientes menores de 60 años (57.24\%), mientras que en el grupo geriátrico fueron las infecciones $(33.96 \%)$ y enfermedades del aparato 


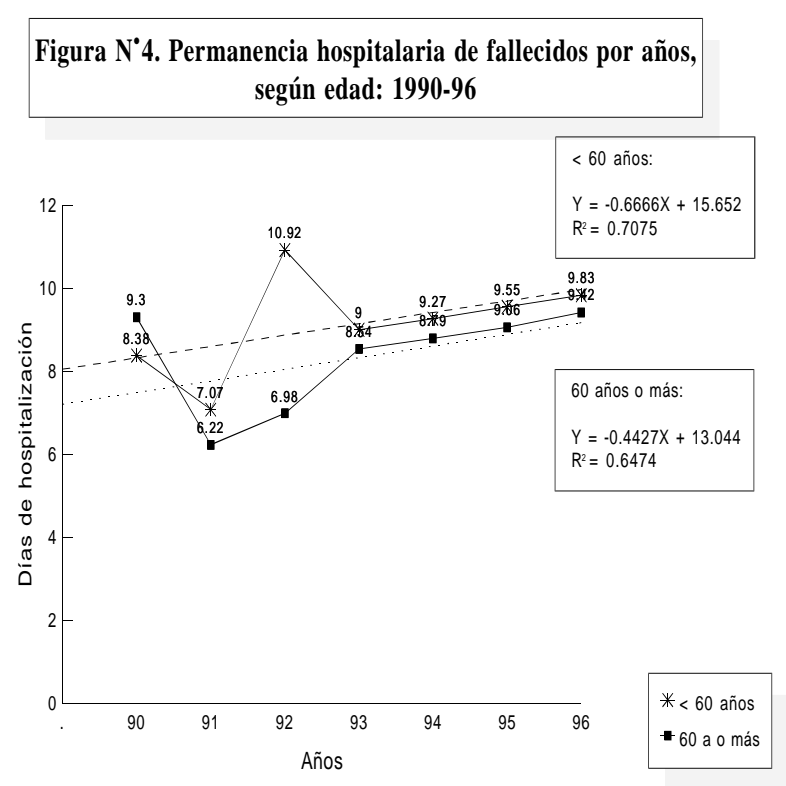

Figura $\mathrm{N}^{\circ}$ 5. Distribución de enfermedades según grupo etáreo: 1990-96.

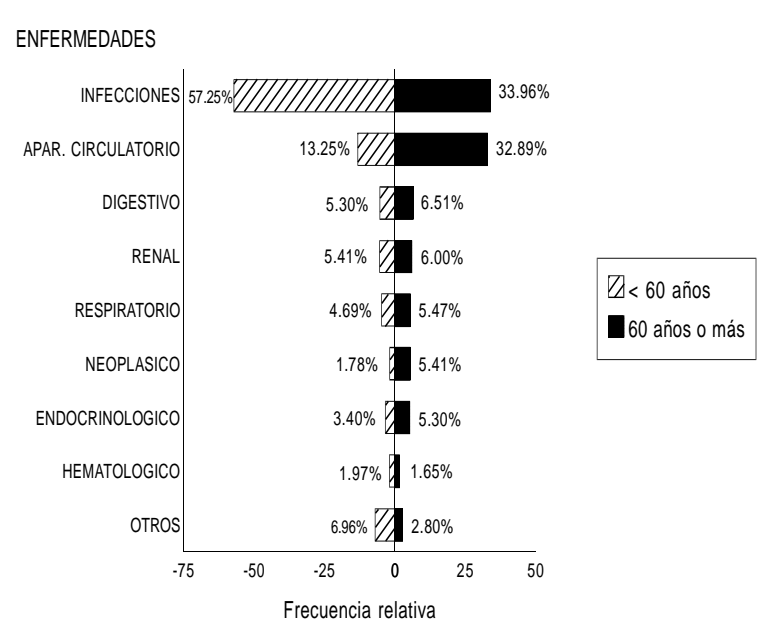

Figura N6. Distribución de mortalidad según edad y sexo: 1990-96.

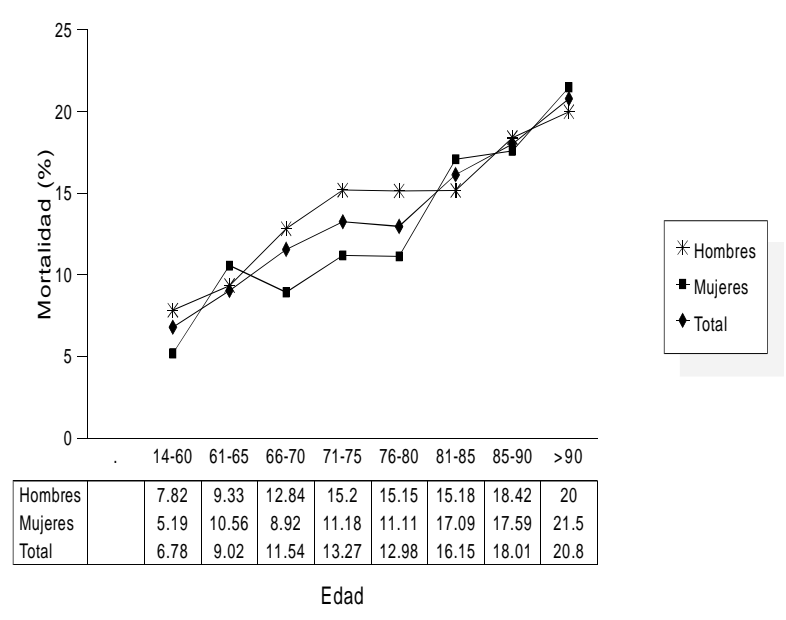

circulatorio (32.89\%). La patología cardiovascular $(18.56 \%)$ y cerebrovascular $(14.33 \%)$, fueron significativamente más frecuentes en los pacientes adultos mayores que en los de menor edad [p<0.01]. (Figura No5).

La mortalidad hospitalaria fue de $12.81 \%$ y $6.79 \%$ para los mayores y menores de 60 años, respectivamente [ $\mathrm{p}<0.01]$; además, fue significativa la mortalidad de los varones mayores $(13.47 \%)$ respecto a las mujeres $(12.11 \%)$ [p<0.01]. En la figura $\mathrm{N}^{\circ} 6$ se muestra un aumento consistente y progresivo de mortalidad a edades mayores, que alcanzó $20.8 \%$ para los mayores de 90 años. También se observa una predominancia en la mortalidad en varones en la mayoría de grupos de edad. En la figura $\mathrm{N}^{\circ} 7$ se puede observar la tendencia progresiva al decrecimiento de mortalidad geriátrica, equivalente a $25 \%$ durante los años consignados; en contraste, los más jóvenes no mostraron cambios significativos.

\section{Consulta externa:}

Durante 1997 fueron atendidos 11080 pacientes de 14 años o más, $16.76 \%$ de los cuales fueron de 60 años de edad o más. Las mujeres constituyeron el $59.74 \%$ y $65.27 \%$ de menores y mayores de 60 años, respectivamente.

La población de pacientes que acudieron más de una vez a consulta externa en el curso de un año, denominados continuadores, estuvo conformada por $53.69 \%$ y $47.36 \%$ de los mayores y menores de 60 años, respectivamente $[\mathrm{p}<0.01]$. Asimismo, fue significativa la diferencia entre los adultos mayores continuadores $(53.69 \%)$ y los que acudieron sólo una vez $(46.31 \%)$ [ $p<0.01]$. En el grupo geriátrico, hubo predominancia de las mujeres continuadoras $(56.68 \%)$ respecto a sus homólogos varones $(48.06 \%)$ [ $p<0.01]$.

La tasa de retorno, índice de las atenciones realizadas por los pacientes continuadores respecto al total de las realizadas en consulta externa, fue $69.23 \%$ y $63.17 \%$ para los mayores y menores de 60 años, respectivamente [p<0.01]. La relación de atenciones/atendidos fue utilizado para revelar el promedio de visitas realizadas por cada paciente en consulta externa. La razón fue de 1.51 y 1.43 para los mayores y menores de 60 años, respectivamente. Dentro del grupo geriátrico, las razones alcanzadas para mujeres y hombres fueron de 1.53 y 1.46 , respectivamente.

En el grupo adulto mayor hubo predominancia de las enfermedades cardiovasculares $(20.14 \%)$ con relación al grupo de menor edad (4.35\%) $[\mathrm{p}<0.01]$ (Figura $\mathrm{N}^{\circ} 8$ ). 


\section{Emergencia:}

Durante 1997 se atendieron 64187 pacientes de 14 años de edad o más, $6.2 \%$ de los cuales conformaron la población de 60 o más años de edad. La patología traumatológica fue la más frecuente en ambos grupos, representando $23.36 \%$ y $24 \%$ de los pacientes geriátricos y de menor edad, respectivamente (Figura $\mathrm{N}^{\circ} 9$ ).

\section{DISCUSION}

A nivel mundial, diversos estudios sobre utilización geriátrica de servicios de salud, han determinado un progresivo incremento en la demanda y utilización de dichos servicios por este sector poblacional $(5,17,18)$. En nuestro medio, muchas de las cifras encontradas años atrás $(19,20,21)$, expresaron la realidad de la atención geriátrica, aunque sin proyectar adecuadamente panoramas de referencia informativa sobre la utilización de servicios geriátricos de salud, necesarios para identificar la vigencia del fenómeno y esbozar estrategias a nivel médico y administrativo.

\section{Hospitalización:}

Nuestra proporción de adultos mayores hospitalizados, es similar a los registros locales $(22,23,24)$ y extranjeros $(5,25,26)$. Hubo mayor representatividad en el grupo de 60 a 69 años, siendo menos numerosos a en los grupos de mayor edad. Esto contrasta con estudios extranjeros, quienes señalan un predominio hospitalario en el grupo de 85 y más años, y un índice de una población geriátrica más añosa $(27,28,29)$. El promedio de edad fue de 73.5 años, superando la esperanza de vida al nacer de 66.74 años para el período 1990-95 (30).

La expectativa de vida nacional, fue de 64.4 y 69.2 años, para hombres y mujeres, respectivamente; esta consideración, en conjunción al significativo predominio de mujeres mayores de 75 años, indica una menor mortalidad y sugieren una mayor sobrevida femenina $(6,21,31)$.

Por otro lado, entre 1990-96, hubo un crecimiento progresivo de adultos mayores hospitalizados, hecho corroborado a nivel local (21) y foráneo (32), sugiriendo así un incremento en la distribución geriátrica hospitalaria a través de los años.

Nuestro promedio de permanencia hospitalaria, para mayores y menores de 60 años, fue de 11.68 y 12.56 días, respectivamente, sugiriendo el alta más precoz de los adultos mayores, tal como lo señalan otros autores locales $(21,23,24)$ y extranjeros $(4,26,27)$. Asimismo, entre
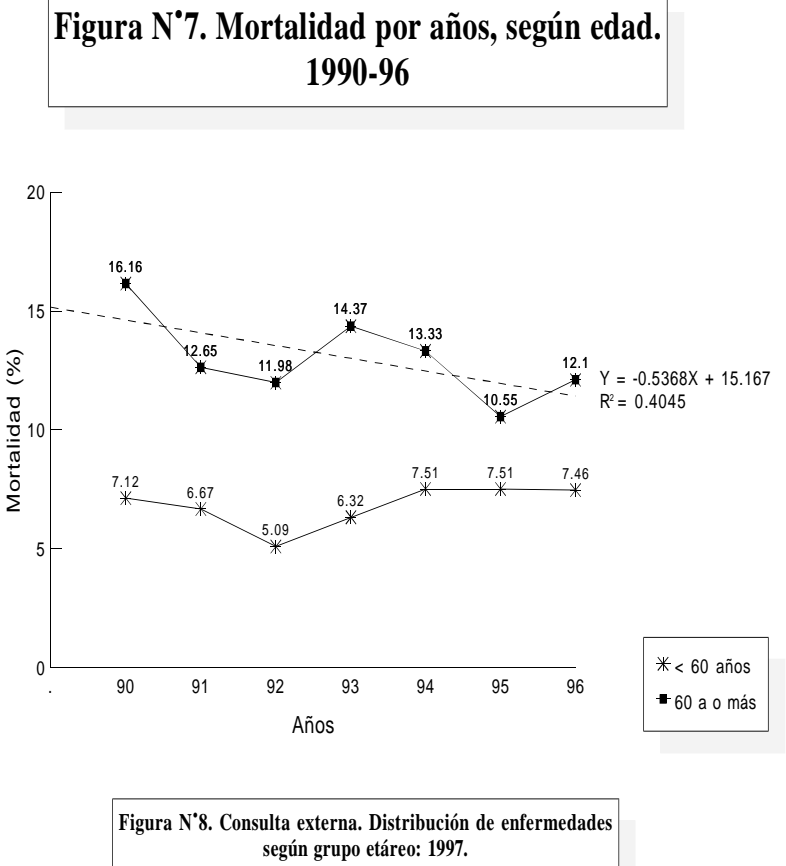
según grupo etáreo: 1997.

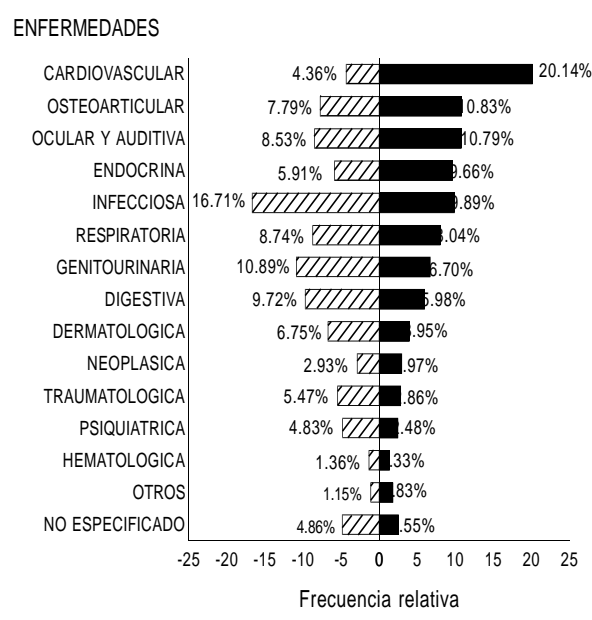

$\bigotimes<60$ años

60 años o más

Figura $\mathrm{N}^{\circ} 9$. Emergencia. Distribución de enfermedades según grupo etáreo: 1997.

\section{ENFERMEDADES}

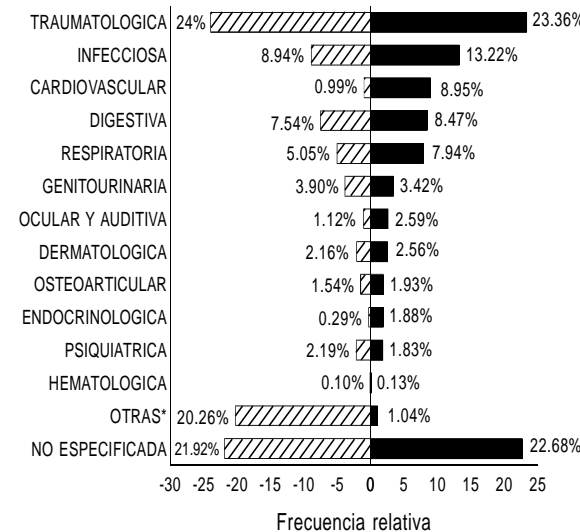

$\nabla><0$ años

60 años o más

* Incluye embarazadas y sus patologías 
los adultos mayores, los de edad más avanzada tuvieron altas más precoces, sugiriendo una actitud particular de manejo ante un usual estado de salud crítico correspondiente a este sector poblacional. Entre 1990-96, hubo una disminución en el promedio de permanencia hospitalaria tanto para los menores como en los mayores de 60 años; actitud consonante con el concepto de alta precoz y sus beneficios desde 1974 (33). Adicionalmente, se observó un aumento del promedio de estadía hospitalaria para los decesos a través de los años, en ambos grupos estudiados.

Con relación a la morbilidad hospitalaria, los adultos mayores mostraron una predominancia compartida entre infecciones y afecciones del aparato circulatorio. Es destacable el gran impacto que aún mantienen las infecciones, tanto en el adulto mayor como en los de menor edad $(1,22,24)$. La prevalencia de enfermedades cardiovasculares y cerebrovasculares, representativos de las afecciones del aparato circulatorio, fueron mayores en la población geriátrica, siendo similar al perfil de los países desarrollados $(34,35,36)$.

La mortalidad geriátrica hospitalaria fue $12.81 \%$ frente a $6.79 \%$ en los menores, diferencia compartida por otros estudios locales $(21,31,37)$. Además, una aproximación de la mortalidad por grupos de edad, reveló una tendencia consistente al incremento conforme aumenta la edad, fenómeno corroborado tanto a nivel nacional (21) como en el extranjero (38).

La mortalidad según sexo muestra en nuestro estudio un índice ligeramente mayor en hombres, diferencia que apoya la mayor supervivencia en 4 años a favor de la mujer en nuestro país (39).

\section{Consulta externa:}

La atención ambulatoria afronta la realidad epidemiológica del adulto mayor, aliviando las formas comunes y a menudo tratables, pero potencialmente discapacitantes y causantes de sufrimiento $(4,40)$. Registros locales revelaron índices de atención menores que el nuestro. Además, encontramos una distribución de mujeres mayores de 60 años que casi duplica la de sus homólogos varones.

Entre los pacientes continuadores se objetivó el predominio de los mayores de 60 años. Entre los mismos adultos mayores, se observó el predominio de los pacientes continuadores sobre quienes realizaron sólo una consulta; resultados que sugieren una necesidad relativamente frecuente de atención en el adulto mayor, dadas las condiciones crónicas predominantes en este grupo de edad.
La tasa de retorno fue superior en el grupo de mayores de 60 años, hecho compartido con estudios locales (21), sugiriendo un mayor sentido de capacidad funcional en consulta externa, hacia la atención de los adultos mayores. Durante 1997 cada paciente mayor y menor de 60 años realizó 1.51 y 1.43 consultas respectivamente, diferencia de 8 atenciones por cada 100 pacientes atendidos, que favorece a los adultos mayores como los usuarios que individualmente realizan más consultas respecto a los de menor edad.

La morbilidad en atención ambulatoria, a diferencia de la dimensión hospitalaria, destaca la real magnitud de problemas que aquejan al adulto mayor, permitiendo una actitud integral de manejo que prolongue su autonomía y optimice su calidad de vida, dado que la prevalencia de enfermedades crónicas es cinco veces mayor que las observadas en los de menor edad (41). En la población geriátrica destacaron, en orden de frecuencia: cardiovasculares, osteoarticulares, y enfermedades oculares y auditivas, panorama similar al hallado en series extranjeras $(41,42,43)$.

\section{Emergencia:}

La morbilidad en emergencia es representativa del estado sanitario y social de la comunidad, con una labor siempre recargada y una capacidad necesariamente rápida para tomar decisiones $(24,44)$. Nosotros encontramos $6.2 \%$ de adultos mayores atendidos en emergencia para 1997, índice superior al hallado por otros autores locales $(19,21)$.

La enfermedad predominante para ambos grupos de edad fueron las traumatológicas, alcanzando a $23.36 \%$ de los adultos mayores atendidos, con un índice de fracturas de $8.9 \%$ respecto al total de eventos traumatológicos, cifras reafirmadas por estudios locales $(45,46)$ y foráneos $(47,48)$. Las infecciones constituyen la segunda condición predominante en ambos grupos de edad, siendo más significativa en el adulto mayor. Este resultado, similar al de otros estudios locales $(19,24)$, es indicio del gran impacto que aún mantiene esta enfermedad, tanto en emergencia como en el resto de servicios, a pesar de la creciente repercusión del fenómeno de transición epidemiológica en nuestro medio $(36,49)$.

Conforme la población avanza en edad, el número de enfermedades crónicas y discapacitantes, así como la consecuente necesidad de atención sanitaria van en aumento. En dicho contexto, el fenómeno demográfico de envejecimiento y los diferentes hallazgos encontrados a lo largo del estudio, confirman la existencia de una significativa utilización de los servicios de 
hospitalización y consulta externa por una creciente población geriátrica usuaria. Es apremiante la elaboración de estrategias adaptadas a su frágil condición fisiológica, que permitan estructurar un marco de atención preventiva, curativa y de rehabilitación al adulto mayor en nuestro medio.

\section{Correspondencia:}

Dr. Luis Varela

Dpto. de Medicina. Hospital Nacional Cayetano Heredia. Universidad Peruana Cayetano Heredia.

Av. Honorio Delgado s/n San Martín de Porres Lima, Perú

\section{BIBLIOGRAFIA}

1. Centro Interamericano de Estudios del Seguro Social. Atención Médico-social a la Tercera Edad. México, 1993; pp. 63 - 73, 81 - 85, 99 - 108.

2. Grundy, E. Mortality and morbidity among the old. BMJ 1984; 288: 663 - 64.

3. Instituto Nacional de Estadística e Informática. Perú: Perfil Socio demográfico de la Tercera Edad. Lima, 1995; pp. $27-37,79-80$.

4. American College of Physicians. Comprehensive functional assessment for elderly patients. Ann Intern Med 1988; 109: 70 - 75.

5. Giaconi, J. Perfil de Morbilidad y Atención en Menores y Mayores de 65 Años. Cuad Med Soc Santiago de Chile. 1985; 26 (3) : 103 - 10.

6. Gonzales, R. El sexo femenino, el sexo fuerte: la evidencia científica. Departamento de Ciencias Fisiológicas. UPCH. Lima, 1996; pp. 176 - 185.

7. Delgado Gamero A. Sistema de Atención Integral de Salud al Anciano. Diagnóstico 1985; 15 (3): 72 - 75.

8. Barrenechea, J. Implicaciones para la Planificación y Administración de los Sistemas de Salud: Salud para Todos el año 2000. Medellín, 1990; pp. 168 - 169, 229 $-232,243-251$.

9. Barturén, A. Psicogeriatría. Diagnostico 1985; 15 (3): $76-78$.

10. Lozano, R. El Peso de la Enfermedad en Adultos Mayores. Salud Pública Mexicana. 1996; 38 (6): 419- 29.

11. Oficina Sanitaria Panamericana. Investigación sobre Servicios de Salud: una Antología. Washington, D.C.: OPS, 1992; pp. 17 - 18.

12. Castañeda, J. Utilización de Servicios de Salud en el Perú. Análisis del Sector Salud: Informe Exploratorio \# 3. Lima: OPS, 1986; pp. $1-10,30-31$.

13. Torres, W. Demanda de Servicios de Salud en el Perú. Análisis del Sector Salud: Informe Exploratorio \# 1. Lima: OPS, 1986; pp. $1-10$.

14. Paganini, J. Análisis de Costos, Demanda y Planificación de Servicios de Salud. Washington, D.C.: OPS. 1990; pp. $222-228,295-299$.

15. Oficina Sanitaria Panamericana. La Atención de los Ancianos: un Desafío para los años 90. Washington, D.C.: OPS, 1994; pp. $3-18,475-84$.
16. Naciones Unidas. Asamblea Mundial Sobre el Envejecimiento: Documento Introductorio. Viena, 1982.

17. Aday, L. The Utilization of Health Services: Indices and Correlates. A Research Bibliography, 1972. Washington, D.C.: National Center for Health Services Research and Development, 1972.

18. Centro Interamericano de Estudios del Seguro Social. El Adulto Mayor en América Latina: sus necesidades y problemas médico-sociales. México, 1995; pp. 1-7, 13 37.

19. Murrieta, R. Unidad o Servicio Geriátrico, Como Parte Integrante del Sistema de Atención Especializado del Paciente Anciano. Tesis de Bachiller en Medicina. UPCH. Lima, 1981.

20. Noriega, M. Hacia una Atención Integral a la Tercera Edad en el Centro Geriátrico Naval. Tesis de Maestría. UPCH. Lima, 1988.

21. Varela L. Estudio comparativo de atención de pacientes mayores y menores de 60 años. Tesis de Especialidad en Medicina Interna. UPCH. Lima, 1992.

22. Alarcón J. Diagnóstico social, nutricional y clínico del anciano hospitalizado. Tesis de Bachiller en Medicina. UPCH.Lima,1989.

23. Benamú, J. Morbimortalidad en Ancianas Hospitalizadas. Tesis de Bachiller en Medicina. UPCH. Lima, 1989.

24. Capuñay, J. Morbimortalidad del Paciente Geriátrico. Tesis de Bachiller en Medicina. UPCH. Lima, 1990.

25. Rai, G. Who should provide hospital care of elderly people. The Lancet. London, 1985; pp. 683 - 85.

26. Rowe, J. Health Care of the Elderly. N Engl J Med 1985; 312 (13): $827-35$.

27. Master, R. A Continuum of Care for the Inner City. N Engl J Med 1980; 302 (26): 1434 - 40.

28. Michel J. Prevalence of infections and their risk factors in geriatric institutions: a one-day multicentre survey. Bulletin of the World Health Organization. Geneva:WHO, 1991. 69 (1): 35 - 41.

29. Winograd, H. Assessment of Geriatric Patients. Scientific American Medicine. USA. 1995; 8: 1 - 8.

30. Instituto Nacional de Estadística e Informática. Proyecciones de Población 1995 - 2025. Lima, 1996; pp. $57-58$.

31. Merino R. Evaluación del paciente geriátrico hospitalizado, orientado por problemas: estudio prospectivo de 71 casos. Tesis de Bachiller en Medicina. UPCH. Lima, 1992.

32. Anzola, E. El Envejecimiento en América Latina y el Caribe. Publicación Científica \# 492: Hacia el Bienestar de los Ancianos. Washington, D.C.: OPS, 1985; pp. 1- 24.

33. Organización Mundial de la Salud. Planeamiento y Organización de los Servicios Geriátricos. Reporte Técnico \# 548. Ginebra: OMS. 1974; pp. 5-13.

34. Fisher, C. Lacunar Strokes and Infarts: a review. Neurology 1982; 32: $871-875$.

35. Ham, R. Atención primaria en geriatría: casos clínicos. USA. 1995; pp. $4-17$.

36. Organización Mundial de la Salud. Informe sobre la Salud en el Mundo 1997. Ginebra: OMS, 1997; pp. 1 -4 . 
37. Rossi, P. Infección Urinaria en Mayores de 60 Años Hospitalizados en el Centro Médico Naval. Tesis de Bachiller en Medicina. UPCH. Lima, 1992.

38. Rubenstein, A. The Sepúlveda GEU Study revised: long - term outcomes use of services, and costs. Aging Milano 1995; 7 (3): 212 - 17.

39. Chu, M. Salud y Autonomía en Relación al Medio Circundante de la Población de 65 y más años en Lima y Cusco. Tesis de Maestría. UPCH. Lima, 1990.

40. Guralnik, J. Disability as a public health outcome in the aging population. Annual Review of Public Health 1996; 17: 25 - 46.

41. Rowe, J. Geriatric Medicine. Boston, 1988; pp 12-22.

42. Fries, J. Aging, Natural death and the Compression of Morbidity. N Engl J Med 1980; 303 (3): 130 - 35.

43. Schwartz, G. Principles and Practice of the Emergency Medicine. USA, 1992; pp. 2559 - 63.

44. Sanders, J. Care of the Elderly in Emergency
Departments: Conclusions and Recommendations. Annals of Emergency Medical 1992; 21 (7): 830 - 34.

45. Gil, J. Caídas en Ancianos Residentes en el Albergue Central Canevaro. Tesis de Bachiller en Enfermería. UPCH. Lima, 1990.

46. Sandoval, L. Caídas en el Adulto Mayor. Estudio de una Serie de Pacientes de Consultorio Externo de Medicina del Hospital Nacional Cayetano Heredia. Rev Med Hered 1996; 7 (3): 119 - 23.

47. Althabe, O. Caídas Accidentales: un Problema de la Tercera Edad. Medical MAG 1997; 2 (21): 54 - 8.

48. Redford, J. Prevención de Caídas en Personas de Edad Avanzada. Tribuna Médica 1992; 85 (4): 147 54.

49. Oficina Sanitaria Panamericana. Perfil de las Condiciones de Salud de los Adultos Mayores de América Latina y el Caribe. Boletín Epidemiológico. Washington, D.C.: OPS, 1997; 18 (2): 11 - 13. 